\title{
Influence of Personal Protective Equipment on Wildland Firefighters' Physiological Response and Performance during the Pack Test
}

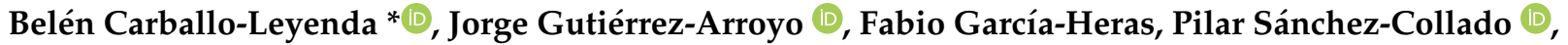 \\ José G. Villa-Vicente (D) and Jose A. Rodríguez-Marroyo *
}

check for updates

Citation: Carballo-Leyenda, B.; Gutiérrez-Arroyo, J.; García-Heras, F.; Sánchez-Collado, P.; Villa-Vicente, J.G.; Rodríguez-Marroyo, J.A. Influence of Personal Protective Equipment on Wildland Firefighters' Physiological Response and

Performance during the Pack Test. Int J. Environ. Res. Public Health 2021, 18, 5050. https://doi.org/10.3390/ ijerph18105050

Academic Editors: Luana Main and Jamie Tait

Received: 7 April 2021

Accepted: 5 May 2021

Published: 11 May 2021

Publisher's Note: MDPI stays neutral with regard to jurisdictional claims in published maps and institutional affiliations.

Copyright: (c) 2021 by the authors. Licensee MDPI, Basel, Switzerland. This article is an open access article distributed under the terms and conditions of the Creative Commons Attribution (CC BY) license (https:// creativecommons.org/licenses/by/ $4.0 /)$.
VALFIS Research Group, Institute of Biomedicine, University of León, 24071 León, Spain; jgutia@unileon.es (J.G.-A.); fgarh@unileon.es (F.G.-H.); mpsanc@unileon.es (P.S.-C.); jg.villa@unileon.es (J.G.V.-V.) * Correspondence: abcarl@unileon.es (B.C.-L.); j.marroyo@unileon.es (J.A.R.-M.)

Abstract: The Pack Test (PT) is a widely used test to establish readiness for work in wildland firefighting. It is common to perform this test dressed in regular exercise clothing. However, wildland firefighters (WFF) have to wear personal protective equipment (PPE) during their deployments, which increases the physiological strain and reduces their work capacity. This study aimed to analyse the impact of full PPE on PT performance. Nine male professional WFF performed in random order a PT walking at the fastest possible self-pace wearing two different clothing configurations: (i) traditional short sports gear (SG) and (ii) the PPE currently used by Spanish WFF. Heart rate (HR), rating of perceived exertion and lap time were recorded during the PT. In addition, oxygen uptake $\left(\mathrm{VO}_{2}\right)$ was estimated through the individual $\mathrm{VO}_{2}-\mathrm{HR}$ relationship previously obtained during a graded exercise test. There was a significant decrease in the PT performance (i.e., completion time) $(\sim 12 \%, p<0.05)$ in PPE. The physiological demands with this configuration were significantly higher $(\sim 10 \%, p<0.05)$. WFF spent $\sim 13 \mathrm{~min}$ above the anaerobic threshold in PPE vs. $\sim 4 \mathrm{~min}$ in SG. A multiple stepwise regression analysis revealed that PT performance variation might be explained by the maximal aerobic velocity $(84.5 \%)$ in PPE and the $\mathrm{VO}_{2 \max }(75.9 \%)$ in SG. In conclusion, wearing complete PPE increases WFF's physiological strain, which translates into a significant PT performance reduction. Performing the test walking at the fastest possible self-pace wearing the PPE would better reflect the high-intensity effort periods reported in real scenarios.

Keywords: wildland firefighting; personal protective equipment; readiness for work; physical employment standards; work capacity

\section{Introduction}

Fighting wildfire is a physically demanding occupation [1,2] requiring firefighters to be physically fit to minimise fatigue and work safely and competently [3]. To determine whether personnel are fit for duty, an increasing number of firefighting agencies (e.g., the USDA Forest Service; Australian Fire Agencies; British Columbia Forest Service in Canada or Ministry of Agriculture, Fisheries and Food in Spain) employ physical competency tests, such as the Pack Test (PT) $[4,5]$. This test involves a $4.83 \mathrm{~km}$ hike over level terrain while carrying a $20.4 \mathrm{~kg}$ pack within $45 \mathrm{~min}$ [6]. The PT was designed to challenge an individual's muscular strength and cardiorespiratory fitness, mimicking the physiological strain encountered during wildland fire suppression using hand tools. While the PT is widely used to assess the subjects' ability to perform sustained and arduous work [7], some aspects of its protocol are not standardised, which might affect its validity and reliability [8]. Specifically, it is common to perform the PT dressed in sports clothing (e.g., shorts, T-shirt, running shoes). However, wildland firefighters (WFF) have to wear their personal protective equipment (PPE) (i.e., coveralls, gloves, helmet and boots) during their deployments [9,10]. This 
fact might affect PT performance since wearing a protective ensemble is heavier $(\sim 6 \mathrm{~kg})$ and more restrictive to heat dissipation than exercise clothing [11,12].

In this context, the effect of the PPE on physiological strain and work capacity has been well documented [10,13-16]. It has been recently reported that wearing WFF's personal protective clothing leads to subjects' decreased performance (i.e., time to exhaustion) of $17 \%$ [10]. Moreover, when other PPE elements such as the helmet, gloves and boots were added, a reduction of $50 \%$ in performance was found [10]. In this regard, the important role of the firefighters' boots has also been previously reported [16]. An increase in exercise demands of approximately $12 \%$ for every $1 \mathrm{~kg}$ of added mass has been shown. To our knowledge, only Phillips et al. [8] have tried to determine the effect of protective equipment on PT performance in healthy subjects. These authors noted that lightweight boots and a fire-resistant cotton coverall might reduce PT performance by $\sim 10 \%$. Researchers pointed out that these elements should be included when assessing WFF's fitness, as they help to replicate the workplace-specific demands. However, the findings of this study might have been conditioned by the subjects' characteristics. A better aerobic capacity $\left(56.2 \pm 0.9 \mathrm{~mL} \cdot \mathrm{kg}^{-1} \cdot \mathrm{min}^{-1}\right)$ has been reported in professional WFF [9]. Additionally, it might be expected that professional WFF would have a greater adaptation to PPE use. On the other hand, in this study, PPE elements such as the helmets, gloves or neck shield were not used, which might have reduced the subjects' physiological strain [10]. Therefore, the present study aimed to analyse the impact of complete personal protective equipment (i.e., coverall, gloves, helmet and boots) on Pack Test performance in professional wildland firefighters.

\section{Material and Methods}

\subsection{Participants}

Nine male professional wildland firefighters participated in this study (Table 1). All of them had more than 7 years of experience as elite wildland firefighters and performed endurance exercise (45-60 min per training session) 3 times per week as part of their scheduled training. Written informed consent was obtained from the participants before starting the study. The test protocol was developed according to the Declaration of Helsinki guidelines for research on human subjects, and it was approved by the Ethics Committee of the University of León, Spain.

Table 1. Anthropometric characteristics and physiological values measured during the graded exercise test.

\begin{tabular}{|c|c|}
\hline Variables & Mean \pm SD \\
\hline Age (years) & $31.1 \pm 3.2$ \\
\hline Body mass $(\mathrm{kg})$ & $82.3 \pm 12.2$ \\
\hline Height $(\mathrm{cm})$ & $177.3 \pm 7.2$ \\
\hline Body surface area $\left(\mathrm{m}^{2}\right)$ & $2.0 \pm 0.2$ \\
\hline $\mathrm{VO}_{2 \max }\left(\mathrm{mL} \cdot \mathrm{kg}^{-1} \cdot \mathrm{min}^{-1}\right)$ & $55.8 \pm 3.6$ \\
\hline Maximal HR (beats. $\min ^{-1}$ ) & $185 \pm 7$ \\
\hline Maximal velocity $\left(\mathrm{km} \cdot \mathrm{h}^{-1}\right)$ & $16.9 \pm 2.6$ \\
\hline $\mathrm{VO}_{2} \mathrm{RCT}\left(\mathrm{mL} \cdot \mathrm{kg}^{-1} \cdot \mathrm{min}^{-1}\right)$ & $45.4 \pm 5.9$ \\
\hline$\% \mathrm{VO}_{2 \max } \mathrm{RCT}$ & $80.8 \pm 4.3$ \\
\hline HR RCT (beats. $\min ^{-1}$ ) & $172 \pm 9$ \\
\hline Velocity RCT $\left(\mathrm{km} \cdot \mathrm{h}^{-1}\right)$ & $14.3 \pm 1.8$ \\
\hline $\mathrm{VO}_{2} \mathrm{VT}\left(\mathrm{mL} \cdot \mathrm{kg}^{-1} \mathrm{~min}^{-1}\right)$ & $36.6 \pm 5.8$ \\
\hline$\% \mathrm{VO}_{2 \max } \mathrm{VT}$ & $64.5 \pm 4.9$ \\
\hline HR VT (beats. $\min ^{-1}$ ) & $140 \pm 7$ \\
\hline Velocity VT $\left(\mathrm{km} \cdot \mathrm{h}^{-1}\right)$ & $10.3 \pm 1.1$ \\
\hline
\end{tabular}

$\mathrm{VO}_{2 \max }$, maximal oxygen uptake; $\mathrm{VT}$, ventilatory threshold; $\mathrm{RCT}$, respiratory compensation threshold; $\% \mathrm{VO}_{2 \max }$, percentage of $\mathrm{VO}_{2 \max }$ at which $\mathrm{VT}$ and $\mathrm{RCT}$ occur. 


\subsection{Experimental Design}

For 2 weeks, subjects performed 3 testing sessions on separate days, with at least $72 \mathrm{~h}$ in between. Participants were asked to refrain from strenuous exercise, excessive sun exposure and alcohol consumption for $48 \mathrm{~h}$ before each experimental session. Volunteers were asked to consume $\sim 250 \mathrm{~mL}$ of water $1 \mathrm{~h}$ before beginning each experimental session [17]. A graded exercise test was performed during the first testing session to determine the subjects' maximal aerobic capacity $\left(\mathrm{VO}_{2 \max }\right)$. During the second and third training sessions, participants performed the PT in random order wearing 2 different clothing configurations: (i) traditional short sports gear (SG) (i.e., shorts, cotton t-shirt, underwear, cotton socks and running shoes) and (ii) the personal protective equipment (PPE) currently used by Spanish WFF (Madrid, Spain), which includes thermal-resistant clothing (65\% fire retardant viscose, 30\% Nomex and $5 \%$ Kevlar, $1.5 \mathrm{~kg}$, surface mass $270 \mathrm{~g} \pm \mathrm{m}^{-2}$, thermal resistance $0.019 \mathrm{~m}^{2} \mathrm{~K} \cdot \mathrm{W}^{-1}$ and evaporative resistance $3.79 \mathrm{~m}^{2} \cdot \mathrm{Pa} \cdot \mathrm{W}^{-1}$ ) and other protective elements such as a helmet, neck shroud, gloves, goggles and midcalf leather boots. In this configuration, SG was worn underneath the PPE.

\subsubsection{Graded Exercise Test}

WFF underwent a graded exercise test on a treadmill ( $\mathrm{h} / \mathrm{p} /$ cosmos pulsar, Cosmos Sports \& Medical GMBH, Nussdorf-Traunstein, Germany). The test started at $6 \mathrm{~km} \cdot \mathrm{h}^{-1}$, and velocity was increased $1 \mathrm{~km} \cdot \mathrm{h}^{-1}$ every $1 \mathrm{~min}$ until volitional exhaustion. The treadmill grade was kept at $1 \%$ throughout the test. Respiratory gas exchange was continuously recorded via a breath-by-breath system (Medisoft Ergocard, Medisoft Group, Sorinnes, Belgium) calibrated following the manufacturer's guidelines. Heart rate (HR) response was also measured with a 12-lead electrocardiogram (Medisoft Medcard, Medi-soft Group, Sorinnes, Belgium). $\mathrm{VO}_{2 \max }$ and maximal $\mathrm{HR}$ were recorded as the highest values obtained for the last $30 \mathrm{~s}$ period before exhaustion. $\mathrm{VO}_{2 \max }$ was considered as valid when at least 2 of the following criteria were reached [18]: $\mathrm{VO}_{2}$ plateau $\left(\leq 150 \mathrm{~mL} \cdot \mathrm{min}^{-1}\right)$, RER $\geq 1.15$, maximal HR was \pm 10 beats of the age-predicted maximal HR (220-age) and rating of perceived exertion (CR $0-10) \geq 8$. The maximal velocity was determined as the highest velocity subjects might maintain for a complete stage, plus the interpolated velocity from incomplete stages [19]. The ventilatory (VT) and respiratory compensation threshold (RCT) were identified according to the following criteria [20]: increase in both ventilation equivalent for oxygen $\left(\mathrm{VE} \cdot \mathrm{VO}_{2}{ }^{-1}\right.$ ) and end-tidal partial pressure of oxygen with no concomitant increase in ventilation equivalent for carbon dioxide $\left(\mathrm{VE} \cdot \mathrm{VCO}_{2}{ }^{-1}\right)$ for $\mathrm{VT}$, an increase in both $\mathrm{VE} \cdot \mathrm{VO}_{2}{ }^{-1}$ and $\mathrm{VE} \cdot \mathrm{VCO}_{2}{ }^{-1}$ and a decrease in end-tidal partial pressure of carbon dioxide for RCT.

\subsubsection{Pack Test}

The PTs were performed on an outdoor $400 \mathrm{~m}$ running track at the same time of day under similar environmental conditions $\left(14.6 \pm 1.9^{\circ} \mathrm{C}\right.$ and $75.0 \pm 14.0 \%$ relative humidity). After a standardized warm-up (15 min of submaximal running and free stretching for $5 \mathrm{~min}$ ), WFF had to complete 12 full laps for a total distance of $4.8 \mathrm{~km}$ in less than $45 \mathrm{~min}$, while carrying a correctly fitted $75 \mathrm{~L}$ backpack weighted to $20.4 \mathrm{~kg}[5-7,21]$. All subjects were instructed to complete the test as fast as possible without running and to stop after completing the required distance. Verbal encouragement was provided throughout the test, but no pacing feedback was administered at any point. Subjects were only informed when half of the test was completed. Throughout the trial, the HR response was measured every $5 \mathrm{~s}$ (RS800, Polar Electro Oy, Kempele, Finland) and was averaged every $30 \mathrm{~s}$ for their analysis. In addition, oxygen uptake $\left(\mathrm{VO}_{2}\right)$ during the PT was estimated through the individual linear relationship between $\mathrm{VO}_{2}$ and $\mathrm{HR}$ obtained during the graded exercise test [22]. Relative $\mathrm{VO}_{2}$ was normalized to total mass, taking into account body mass and load carriage (i.e., backpack and PPE mass) (Taylor et al., 2012). Rating of perceived exertion (RPE) was obtained during the last $10 \mathrm{~m}$ of every lap using the Borg scale (1-10) [23]. A photocell timing system (DSD Laser System, DSD Inc., León, Spain) was used to measure 
the total time and lap time. Finally, walking cadence was calculated by visually counting the number of steps in $1 \mathrm{~min}$ in the middle course of laps 1,6 and 12 [24]. All subjects were previously familiar with the use of the PT.

\subsection{Data Analysis}

The results are expressed as mean \pm standard deviation (SD). The assumption of normality was verified using the Shapiro-Wilk's test. A paired student's $t$-test was applied to establish differences in PT completion time and walking cadence. The variables analysed throughout the PT (i.e., lap time, $\mathrm{HR}, \% \mathrm{HR}_{\max }, \mathrm{VO}_{2}$ and $\mathrm{RPE}$ ) were compared using a 2-way ANOVA with repeated measures (clothing configuration $\times$ time). When a significant $F$-value was found, Bonferroni's post hoc comparison was used to establish significant differences between means. The assumption of sphericity was checked using Mauchly's test, and if this assumption was violated, the Greenhouse-Geisser adjustment was performed. Cohen's $d$ and partial eta-squared $\left(\eta_{\mathrm{p}}{ }^{2}\right)$ were calculated as a measure of effect size. Cohen's $d$ values of $<0.20,0.20-0.50,0.51-0.80$ and $>0.80$ were rated as trivial, small, moderate and large effects, respectively [25]. When $\left(\eta_{\mathrm{p}}{ }^{2}\right)$ was reported, values of $0.01,0.06$ and 0.14 were considered small, moderate and large, respectively [26]. The relationship between variables was determined using the Pearson correlation coefficient $(r)$. Magnitudes of $<0.1,<0.4,<0.6$, $<0.9$ and $>0.9$ were classified as trivial, small, moderate, strong, very strong and almost perfect, respectively [27]. Finally, a multiple stepwise regression analysis was used to establish a predictive equation to estimate subjects' PT performance from the submaximal and maximal variables assessed in the graded exercise test. Collinearity tolerance statistics were calculated to determine the correlation between predictor variables. Any variable that had a tolerance level of less than 0.10 was not included in the model. Values of $p<0.05$ were considered statistically significant. Analyses were performed using SPSS+ V.25.0 statistical software (SPSS, Inc., Chicago, IL, USA).

\section{Results}

The results of the anthropometric and graded exercise tests performed in the laboratory that define the WFF's morphological and cardiopulmonary characteristics are shown in Table 1. There was a significant decrease in PT performance $(p<0.05, d=0.94)$ when WFF wore PPE (Table 2). A significant $(p<0.05)$ interaction effect between clothing configuration and time was analysed for lap time. From the fifth time lap on, significant differences $\left(p<0.05, \eta_{\mathrm{p}}{ }^{2}=0.45\right)$ between PPE and SG were obtained (Figure 1). Physiological demands were significantly higher $(p<0.05)$ in PPE configuration (Table 2$)$. In addition, an interaction effect $(p<0.05)$ between clothing configuration and time on $\mathrm{HR}$ and $\mathrm{VO}_{2}$ was obtained. Both the $\mathrm{HR}$ and estimated $\mathrm{VO}_{2}$ were higher with PPE than SG $\left(p<0.05, \eta_{\mathrm{p}}^{2}=0.73,0.71\right)$ throughout the PT (Figure 2). However, no significant differences between conditions in RPE or walking cadence were found (Table 2). Despite this, the magnitude of the differences in the RPE was moderate. Finally, all variables showed a significantly increase over the PT course $\left(p<0.05, \eta_{\mathrm{p}}^{2}=0.54-0.99\right)$ (Figures 1 and 2).

Table 2. Mean performance, physiological and perceptual responses (mean $\pm \mathrm{SD}$ ) during the Pack Test performed wearing short sports gear (SG) and personal protective equipment (PPE).

\begin{tabular}{|c|c|c|c|}
\hline & SG & PPE & Cohen's $d$ (rating) \\
\hline Pack Test completion time (min) & $33.3 \pm 3.9$ & $37.2 \pm 4.4$ * & 0.94 (large) \\
\hline Heart rate (beats $\cdot \mathrm{min}^{-1}$ ) & $157 \pm 9$ & $169 \pm 8^{*}$ & 1.46 (large) \\
\hline Percentage of maximal HR (\%) & $84.4 \pm 4.4$ & $89.7 \pm 5.1 *$ & 1.06 (large) \\
\hline Estimated oxygen uptake $\left(\mathrm{L} \cdot \mathrm{min}^{-1}\right)$ & $3.2 \pm 0.4$ & $3.6 \pm 0.5 *$ & 1.06 (large) \\
\hline Estimated oxygen uptake $\left(\mathrm{mL} \cdot \mathrm{kg}_{\text {total mass }}{ }^{-1} \cdot \mathrm{min}^{-1}\right)$ & $33.1 \pm 5.6$ & $35.8 \pm 4.9 *$ & 0.51 (moderate) \\
\hline Percentage of $\mathrm{VO}_{2 \max }(\%)$ & $61.0 \pm 4.4$ & $66.6 \pm 4.2 *$ & 1.34 (large) \\
\hline Rating of perceived exertion & $6.8 \pm 0.6$ & $7.4 \pm 1.1$ & 0.68 (moderate) \\
\hline Walking cadence (steps $\cdot \mathrm{min}^{-1}$ ) & $155 \pm 17$ & $155 \pm 17$ & 0.00 (trivial) \\
\hline
\end{tabular}

\footnotetext{
* Significant difference $(p<0.05)$.
} 


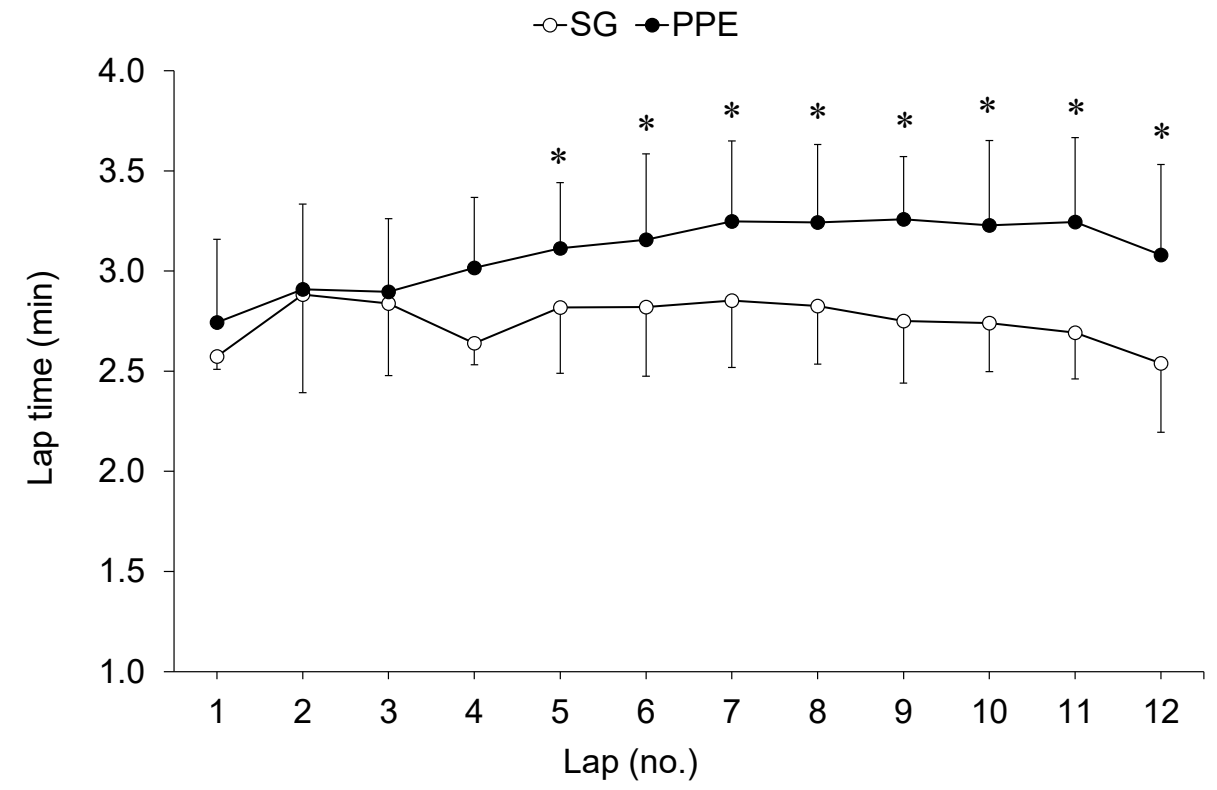

Figure 1. Lap time during the Pack Test performed wearing traditional short sports gear (SG) and wildland firefighters' personal protective equipment (PPE). Values are mean $\pm \mathrm{SD}$. * indicates significant difference $(p<0.05)$.

A

$\rightarrow-S G \quad-P P E$
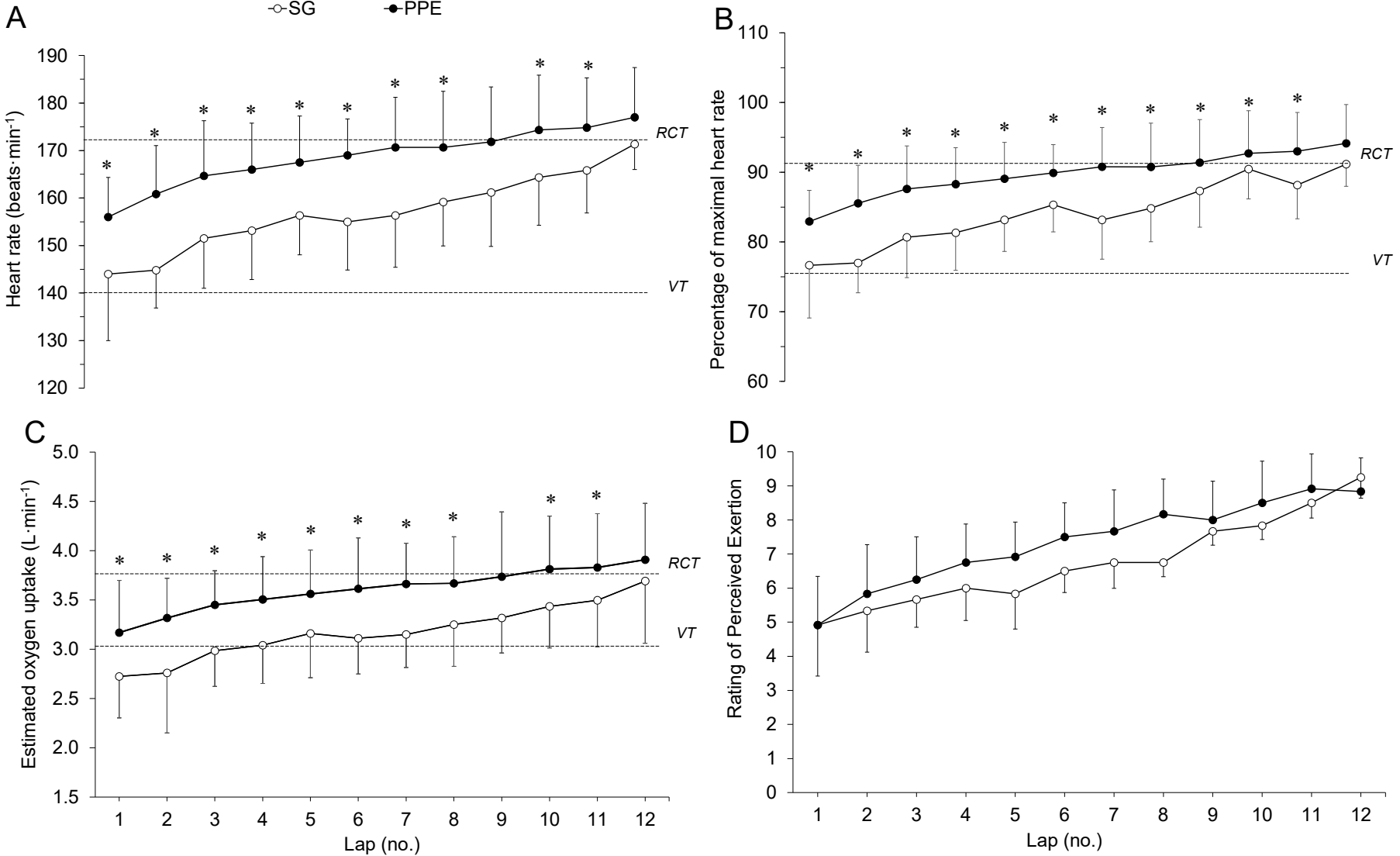

Figure 2. Heart rate $(\mathbf{A}, \mathbf{B})$, estimated $\mathrm{VO}_{2}(\mathbf{C})$ and rating of perceived exertion (D) throughout the Pack Test wearing traditional short sports gear (SG) and a wildland firefighters' personal protective equipment (PPE). RCT, respiratory compensation threshold; VT, ventilatory threshold. Values are mean \pm SD. ${ }^{*}$ indicates significant difference $(p<0.05)$.

There was a strong relationship between PT performance in SG and PPE $(r=0.90$, $p<0.05)$. Furthermore, the PT performance was correlated with $\mathrm{VO}_{2 \max }$, maximal velocity 
and velocity at RCT in SG ( $r=-0.90,-0.89$ and -0.82 , respectively) and PPE ( $r=-0.91$, -0.94 and -0.86 , respectively). The multiple regression analysis revealed that $84.5 \%$ of the PT performance variation might be explained by the maximal aerobic velocity reached in the graded exercise test in PPE ( $\mathrm{y}=60.89-1.396$ maximal velocity, $p<0.01$, SEE $=1.54 \mathrm{~min}$ ). Conversely, $75.9 \%$ of the PT performance variation might be explained by the $\mathrm{VO}_{2 \max }$ in $\mathrm{SG}\left(\mathrm{y}=53.623-0.397 \mathrm{VO}_{2 \max }, p<0.05, \mathrm{SEE}=1.73 \mathrm{~min}\right)$.

\section{Discussion}

The main finding of this study was that the use of PPE increased the WFF's physiological strain $(\sim 10 \%)$, leading to a significant reduction in PT performance $(\sim 12 \%)$ versus SG. These results highlight the influence that PPE has on the exercise demands during the PT, which should be taken into account when administering this test to specifically assess the WFF's physical competency. Overall, these results are consistent with those previously reported $[10,16,28,29]$, which demonstrate the negative impact on performance when exercising in occupational protective ensembles. The higher physiological demand for PPE (Figure 2) might have been a consequence of the mass difference $(6.8 \pm 1.4 \mathrm{~kg})$ between PPE and SG $[16,28,29]$. In this regard, increases in $\mathrm{VO}_{2}$ of $2.7 \%$ per $\mathrm{kg}$ of clothing mass has been previously reported [28]. In addition, mass distribution might have played an important role. In this sense, Taylor et al. [16] showed the importance of the firefighting boots' mass $(\sim 2.5 \mathrm{~kg})$ in increasing metabolic cost $(\sim 11 \%)$. Therefore, it seems plausible to think that the WFF's boots in this study $(\sim 2.0 \mathrm{~kg})$ might have contributed to the increased $\mathrm{HR}$ and estimated $\mathrm{VO}_{2}$ (Figure 2).

Although the increase of the mass in PPE might mainly explain the increase in the metabolic rate analysed, the use of the protective suit involves wearing an additional layer, which might suppose a worsened economy of movement [8]. When the estimated $\mathrm{VO}_{2}$ was normalised to total mass, a significantly higher value in PPE was found (Table 2). Earlier studies have reported a $3 \%$ increase in $\mathrm{VO}_{2}$ for each additional layer worn during wholebody exercise [30,31]. Even a reduction in the subjects' mobility caused by the PPE elements might contribute to this fact by causing a negative effect on PT walking velocity [32].

On the other hand, performing the PT with PPE might lead to greater thermal stress $[33,34]$. Wearing PPE has been associated with a higher cardiovascular strain due to the body heat dissipation restriction $[10,13,14]$. This fact forces the thermoregulatory system to increase the cutaneous blood flow in an attempt to enhance body heat release $[10,13,14]$. In this study, the thermal state between conditions could not be discussed since the core temperature was not measured. However, it may be speculated that the higher HR in PPE ( 12 bpm) might, in part, be an added effect of a higher body heat storage as a result of the decrease in the exposed skin surface, reducing heat exchange with the ambient $[12,35]$. Recently, the role of wearing complete PPE vs. sports clothing on WFF's thermophysiological response has been reported [10]. Results of Carballo-Leyenda et al. [10] showed that PPE might significantly worsen WFF's performance and substantially increase physiological demands $(22 \%)$ compared to a sports gear ensemble due to both the mass increase and body heat storage.

The findings of the present study substantiate those reported by Phillips et al. [8] These authors informed of a decrease in PT performance of $\sim 3 \%$ when healthy subjects wore protective clothing and boots. However, the negative impact of PPE on PT performance was four times higher in our study $(\sim 12 \%)$. Similarly, the effect that PPE had on HR was greater in our WFF sample ( $8 \%$ vs. $4 \%$ ). These differences might be related to the fact that our study subjects performed the test completely encapsulated, while subjects in Phillips et al. [8] only wore protective clothing and work boots. Several studies have reported an increase in thermal and cardiovascular strain when covering the head with a helmet, mask or hood $[10,16,35,36]$. Specifically, the relevance of the PPE elements has been examined. Carballo-Leyenda et al. [10] have shown that adding the helmet, boots and gloves to the fire-resistant coverall may reduce WFF's performance by $50 \%$ and increase the metabolic demand by $20 \%$ vs. wearing only the protective suit. Despite this, PT performance was better $(\sim 12 \%)$ in our study subjects, possibly because of their better aerobic capacity ( $\sim 56 \mathrm{vs.}$ 
$\left.\sim 47 \mathrm{~mL} \cdot \mathrm{kg}^{-1} \cdot \mathrm{min}^{-1}\right)$. Indeed, the PT performance with a similar configuration (SG) was $\sim 19 \%$ better in our subjects. We found a strong relationship $(r<-0.90)$ between aerobic capacity parameters (e.g., $\mathrm{VO}_{2 \max }$ and maximal aerobic velocity) and PT performance both in PPE and SG. In addition, the multiple regression analysis revealed that most of the variation in PT performance might be explained by the maximal aerobic velocity $(84.5 \%)$ in PPE and the $\mathrm{VO}_{2 \max }(75.9 \%)$ in SG. These results seem to indicate that neuromuscular and anaerobic characteristics might have greater importance in PT performance when the WFF wore PPE [37].

All WFF completed the PT below the cut-score of $45 \mathrm{~min}$ (Table 2). Originally, this time was established based on the linear regression between PT total time and $\mathrm{VO}_{2 \max }$, associated with a minimum requirement for WFF of $45 \mathrm{~mL} \cdot \mathrm{kg}^{-1} \cdot \mathrm{min}^{-1}$ [7]. Taking our data into account, a PT time of $45 \mathrm{~min}$ equated to a $\mathrm{VO}_{2 \max }$ of $\sim 27$ and $\sim 34 \mathrm{~mL} \cdot \mathrm{kg}^{-1} \cdot \mathrm{min}^{-1}$ for SG and PPE, respectively. This discrepancy was likely due to the fact that the subjects of this study walked at a self-selected velocity faster than the pace required to complete the test in $45 \mathrm{~min}$. Indeed, the PT estimated $\mathrm{VO}_{2}\left(\sim 39\right.$ and $\sim 44 \mathrm{~mL} \cdot \mathrm{kg}^{-1} \cdot \mathrm{min}^{-1}$ in SG and PPE, respectively) was substantially higher than that previously reported $\left(22.2 \mathrm{~mL} \cdot \mathrm{kg}^{-1} \cdot \mathrm{min}^{-1}\right)$ [21] The mean exercise intensity analysed in PPE and SG was higher (Table 2) than those observed during real wildland fire suppressions in professional WFF [9,38]. This was mainly because of the different character of the effort performed during the PT and wildfire firefighting (continuous vs. intermittent). However, when WFF performed the PT wearing PPE, the time spent above the RCT ( 13 and $\sim 4$ min in PPE and SG, respectively) was similar to that observed in WFF during their deployments $(\sim 10 \mathrm{~min})[9,38]$. These results provide additional evidence on the role of PPE and self-selected pace in PT performance and how this fact might be of paramount relevance when assessing physiological readiness for work. A work readiness assessment should help evaluate WFF's capacity to tolerate the highest work intensities and heat strain associated with wearing PPE [8,29]. It has been suggested that $15 \%$ of new WFF recruits who meet the PT pass scores would have difficulty performing their tasks while wearing PPE [12].

The present study presents some potential limitations. First, $\mathrm{VO}_{2}$ values were estimated. This might restrict the study validity since different factors such as circadian rhythms and thermal stress might have influenced the results [39]. Nevertheless, environmental conditions during the testing sessions and the fact that all tests were performed at the same time of day might have controlled the influence of these factors. Furthermore, it has been widely established that there is a strong relationship between $\mathrm{HR}$ and $\mathrm{VO}_{2}$ within subjects $[22,40]$. Second, the core temperature was not measured. Its measurement might have provided information about the subjects' thermal strain and heat storage contribution to the physiological and perceptive response. In this regard, the HR reflected the combined load that the exercising muscles and thermoregulatory demands posed on the cardiovascular system. Therefore, the magnitude of the changes observed in this study between configurations may provide insight into the repercussions of wearing the PPE. Finally, the study did not consider female WFF, which may affect the obtained results. In this regard, additional research effort is required to determine if the findings reported in this study are applicable to females.

\section{Conclusions}

Findings from this study show that wearing complete personal protective equipment increases the professional WFF's physiological strain, which translates into a significant reduction in PT performance. Our results highlight the requirement for the inclusion of personal protective equipment when assessing physiological readiness for work. In addition, it is advisable to perform the test by walking at the fastest possible self-pace. All this might contribute to achieving a high exercise demand, similar to those performed by the WFF in the hardest periods of work during wildfire suppression. Future studies should delve into the suitability of establishing a cut-off point of $45 \mathrm{~min}$ in this test. 
Author Contributions: Conceptualization, B.C.-L., J.G.-A. and F.G.-H.; methodology and data collection, B.C.-L., J.G.-A. and F.G.-H.; formal analysis, B.C.-L. and J.A.R.-M.; writing-original draft preparation, B.C.-L. and J.A.R.-M.; writing-review and editing, B.C.-L., J.A.R.-M., P.S.-C. and J.G.V.-V. All authors have read and agreed to the published version of the manuscript.

Funding: This research received no external funding.

Institutional Review Board Statement: The study was conducted according to the guidelines of the Declaration of Helsinki and approved by the Ethics Committee of the University of León (025-2020, 22 July 2020).

Informed Consent Statement: Informed consent was obtained from all subjects involved in the study.

Data Availability Statement: The data presented in this study are available on request from the corresponding author. The data are not publicly available due to privacy restrictions.

Conflicts of Interest: The authors declare no conflict of interest.

\section{References}

1. Cuddy, J.S.; Sol, J.A.; Hailes, W.S.; Ruby, B.C. Work patterns dictate energy demands and thermal strain during wildland firefighting. Wilderness Environ. Med. 2015, 26, 221-226. [CrossRef]

2. Ruby, B.C.; Shriver, T.C.; Zderic, T.W.; Sharkey, B.J.; Burks, C.; Tysk, S. Total energy expenditure during arduous wildfire suppression. Med. Sci. Sports Exerc. 2002, 34, 1048-1054. [CrossRef]

3. Sharkey, B.; Rothwell, T.; DeLorenzo-Green, T. Development of a job-related work capacity test for wildland firefighters. Med. Sci. Sports Exerc. 1994, 26, S88. [CrossRef]

4. Sharkey, B. Fitness and Work Capacity; USDA Forest Service, Missoula Technology and Development Centre: Missoula, MT, USA, 1997.

5. Petersen, A.; Payne, W.; Phillips, M.; Netto, K.; Nichols, D.; Aisbett, B. Validity and relevance of the pack hike wildland firefighter work capacity test: A review. Ergonomics 2010, 53, 1276-1285. [CrossRef] [PubMed]

6. Sharkey, B.J. Wildland Firefighter Health and Safety: Recommendations of the April 1999 Conference; USDA Forest Service, Missoula Technology and Development Centre: Missoula, MT, USA, 1999. Available online: https://www.fs.fed.us/t-d/pubs/pdfpubs/ pdf99512841/pdf99512841pt03.pdf (accessed on 25 February 2021).

7. Sharkey, B.; Rothwell, T. Validation and field evaluation of a work capacity test for wildland firefighters. Med. Sci. Sports Exerc. 1996, 28, S79. [CrossRef]

8. Phillips, D.B.; Ehnes, C.M.; Welch, B.G.; Lee, L.N.; Simin, I.; Petersen, S.R. Influence of work clothing on physiological responses and performance during treadmill exercise and the Wildland Firefighter Pack Test. Appl. Ergon. 2018, 68, 313-318. [CrossRef]

9. Rodríguez-Marroyo, J.A.; López-Satue, J.; Pernía, R.; Carballo, B.; García-López, J.; Foster, C.; Villa, J.G. Physiological work demands of Spanish wildland firefighters during wildfire suppression. Int. Arch. Occup. Environ. Health 2012, 85, 221-228. [CrossRef] [PubMed]

10. Carballo-Leyenda, B.; Villa, J.G.; López-Satué, J.; Collado, P.S.; Rodríguez-Marroyo, J.A. Fractional contribution of wildland firefighters' personal protective equipment on physiological strain. Front. Physiol. 2018, 9, 1139. [CrossRef] [PubMed]

11. McLellan, T.M.; Daanen, H.A.M.; Cheung, S.S. Encapsulated environment. Compr. Physiol. 2013, 3, 1363-1391. [CrossRef] [PubMed]

12. Mclellan, T.M.; Havenith, G. Protective clothing ensembles and physical employment standards. Appl. Physiol. Nutr. Metab. 2016, 41, 121-130. [CrossRef] [PubMed]

13. Smith, D.L.; Petruzzello, S.J.; Kramer, J.M.; Warner, S.E.; Bone, B.G.; Misner, J.E. Selected physiological and psychobiological responses to physical activity in different configurations of firefighting gear. Ergonomics 1995, 38, 2065-2077. [CrossRef]

14. Selkirk, G.A.; McLellan, T.M. Physical Work Limits for Toronto Firefighters in Warm Environments. J. Occup. Environ. Hyg. 2004, 1, 199-212. [CrossRef] [PubMed]

15. Dreger, R.W.; Jones, R.L.; Petersen, S.R. Effects of the self-contained breathing apparatus and fire protective clothing on maximal oxygen uptake. Ergonomics 2006, 49, 911-920. [CrossRef] [PubMed]

16. Taylor, N.A.S.; Lewis, M.C.; Notley, S.R.; Peoples, G.E. A fractionation of the physiological burden of the personal protective equipment worn by firefighters. Eur. J. Appl. Physiol. 2012, 112, 2913-2921. [CrossRef] [PubMed]

17. Sawka, M.N.; Burke, L.M.; Eichner, E.R.; Maughan, R.J.; Montain, S.J.; Stachenfeld, N.S. American College of Sports Medicine position stand. Exercise and fluid replacement. Med. Sci. Sports Exerc. 2007, 39, 377-390. [CrossRef]

18. Midgley, A.W.; Carroll, S.; Marchant, D.; McNaughton, L.R.; Siegler, J. Evaluation of true maximal oxygen uptake based on a novel set of standardized criteria. Appl. Physiol. Nutr. Metab. 2009, 34, 115-123. [CrossRef] [PubMed]

19. Kuipers, H.; Verstappen, F.T.J.; Keizer, H.A.; Geurten, P.; van Kranenburg, G. Variability of aerobic performance in the laboratory and its physiologic correlates. Int. J. Sports Med. 1985, 6, 197-201. [CrossRef]

20. Davis, J.A. Anaerobic threshold: Review of the concept and directions for future research. Med. Sci. Sports Exerc. 1985, 17, 6-21. [CrossRef] 
21. DeLorenzo-Green, T.; Sharkey, B.J. Development and validation of a work capacity test for wildland firefighters. Med. Sci. Sports Exerc. 1995, 27, S166. [CrossRef]

22. Astrand, P.O.; Rodahl, K.; Dahl, H.; Stromme, S. Textbook of Work Physiology; Human Kinetics: Champaign, IL, USA, 2003.

23. Borg, G.A. Psychophysical bases of perceived exertion. Med. Sci. Sports Exerc. 1982, 14, 377-381. [CrossRef] [PubMed]

24. Tudor-Locke, C.; Aguiar, E.J.; Han, H.; Ducharme, S.W.; Schuna, J.M.; Barreira, T.V.; Moore, C.C.; Busa, M.A.; Lim, J.; Sirard, J.R.; et al. Walking cadence (steps $/ \mathrm{min}$ ) and intensity in 21-40 year olds: CADENCE-adults. Int. J. Behav. Nutr. Phys. Act. 2019, 16, 8. [CrossRef]

25. Cohen, J. Statistical Power Analysis for the Behavioral Sciences, 2nd ed.; Lawrence Erlbaum Associates Publishers: New York, NY, USA, 1988; Available online: http://www.utstat.toronto.edu/ \{\}brunner/oldclass/378f16/readings/CohenPower.pdf (accessed on 12 February 2021).

26. Lakens, D. Calculating and reporting effect sizes to facilitate cumulative science: A practical primer for $t$-tests and ANOVAs. Front. Psychol. 2013, 4, 863. [CrossRef] [PubMed]

27. Hopkins, W.G. A New View of Statistics. 2006. Available online: https://www.sportsci.org/resource/stats/effectmag.html (accessed on 15 January 2021).

28. Dorman, L.E.; Havenith, G. The effects of protective clothing on energy consumption during different activities. Eur. J. Appl. Physiol. 2009, 105, 463-470. [CrossRef]

29. Lee, J.-Y.; Bakri, I.; Kim, J.-H.; Son, S.-Y.; Tochihara, Y. The impact of firefighter personal protective equipment and treadmill protocol on maximal oxygen uptake. J. Occup. Environ. Hyg. 2013, 10, 397-407. [CrossRef]

30. Teitlebaum, A.G.R. Increased energy cost with multiple clothing layers. J. Appl. Physiol. 1979, 32, 742-744. [CrossRef] [PubMed]

31. Duggan, A. Energy cost of stepping in protective clothing ensembles. Ergonomics 1988, 31, 3-11. [CrossRef] [PubMed]

32. Coca, A.; Williams, W.J.; Roberge, R.J.; Powell, J.B. Effects of fire fighter protective ensembles on mobility and performance. Appl. Ergon. 2010, 41, 636-641. [CrossRef]

33. Bruce-Low, S.S.; Cotterrell, D.; Jones, G.E. Effect of wearing personal protective clothing and self-contained breathing apparatus on heart rate, temperature and oxygen consumption during stepping exercise and live fire training exercises. Ergonomics 2007, 50, 80-98. [CrossRef] [PubMed]

34. Cuddy, J.S.; Hailes, W.S.; Ruby, B.C. A reduced core to skin temperature gradient, not a critical core temperature, affects aerobic capacity in the heat. J. Therm. Biol. 2014, 43, 7-12. [CrossRef]

35. Lee, J.-Y.; Kim, S.; Jang, Y.-J.; Baek, Y.-J.; Park, J. Component contribution of personal protective equipment to the alleviation of physiological strain in firefighters during work and recovery. Ergonomics 2014, 57, 1068-1077. [CrossRef]

36. Montain, S.J.; Sawka, M.N.; Cadarette, B.S.; Quigley, M.D.; McKay, J.M. Physiological tolerance to uncompensable heat stress: Effects of exercise intensity, protective clothing, and climate. J. Appl. Physiol. 1994, 77, 216-222. [CrossRef]

37. Paavolainen, L.; Nummela, A.; Rusko, H. Muscle power factors and $\mathrm{VO}_{2 \max }$ as determinants of horizontal and uphill running performance. Scand. J. Med. Sci. Sports 2000, 10, 286-291. [CrossRef] [PubMed]

38. Rodríguez-Marroyo, J.A.; Villa, J.G.; López-Satue, J.; Pernía, R.; Carballo, B.; García-López, J.; Foster, C. Physical and thermal strain of firefighters according to the firefighting tactics used to suppress wildfires. Ergonomics 2011, 54, 1101-1108. [CrossRef] [PubMed]

39. Achten, J.; Jeukendrup, A.E. Heart Rate Monitoring. Sports Med. 2003, 33, 517-538. [CrossRef] [PubMed]

40. Wicks, J.R.; Oldridge, N.B.; Nielsen, L.K.; Vickers, C.E. HR Index-A Simple Method for the Prediction of Oxygen Uptake. Med. Sci. Sports Exerc. 2011, 43, 2005-2012. [CrossRef] 